\title{
The Help and Influence of Memetics Theory in Applied Linguistic on Second Language Acquisition Teaching
}

\author{
Lin $\mathrm{Na}^{1, \mathrm{a}}$ \\ ${ }^{1}$ Xi'an Peihua University, Xi'an, Shaanxi, 710125 \\ ${ }^{\mathrm{a}}$ email
}

Keywords: University English, Memetics Theory, Acquisition Teaching

\begin{abstract}
College English teaching has a history more than ten years in our country, focusing on only from the transmission of language knowledge to improve the use of language skills. Through the test results of the past few years, it can be clearly found in a variety of skills, the weakest part of the students is writing, so how to improve the level of students in English writing has become the hot spots of a large number of English teaching experts and scholars. On the basis of the research results of English writing at home and abroad, this paper combines the meme theory and the second language acquisition theory, and applies these theories to English writing teaching. It is proved that the meme theory has a good effect on English writing, which can effectively improve the students' English writing level.
\end{abstract}

\section{Introduction}

Memetics is based on the Darwinian theory of evolution, used to explain the evolution of the law of a new theory. A memo is an information unit that is copied and propagated by imitation. Template refers to the culture of people in the field of imitation between each other, spread the idea or idea, and passed down from generation to generation. It is closely linked with the human culture, and the spread of human culture is mainly by language. Imitation is the basic feature of the memo and the core content of the copy, but also the source of the key to spread. The scope of the use of meme is becoming more and more widely used and more and more disciplines are applied to the modal theory. At the same time, the study of memetics has produced several influential major schools, such as the concept of memes, cultural evolution and so on. In China, some important scholars how natural and so on, the introduction of the theory of memetics to linguistic research and to promote, use, broaden the new field of linguistics research.

As a carrier of cultural communication, Language also reflects the cultural phenomenon. In the study of language, memetics plays an increasingly important role, especially in the study of verbal communication and social evolution. The language itself is a meme. The language will become a meme at different levels, such as words, passages, chapters, etc., and the way it needs is to imitate and spread. In the process of imitation and propagation, the language model will combine with different contexts to produce new memes. The model is favorable to the development of language. Both sides of the communication in the use of language is according to the specific context and communication purposes to imitate, integration, which prompted the emergence of new modal variants.

The philosophy of language embodies the law of discourse reproduction and dissemination from the perspective of memetics. There are two ways to copy and spread the language: genotype and phenotype. The former refers to the information in different ways to copy and spread, the latter refers to the form of the same and different content of the spread of the way. The memo theory has the characteristics of imitation, reproducibility and formal diversity. Heylighen divides the replication of the memes into four stages. The first stage is assimilation. It is the first reason that should be exposed to the new individual and its understanding, acceptance. The second stage is memory. It is the retention time of the memes in memory. Memory is selective, only a small number of molds can be preserved. The third stage is to express. It refers to the mold because of the exchange with other individuals from the memory storage model library expressed. The fourth stage 
is transmission. It requires that the vector of the expressed vector has stability. At present, the study of memetics is focused on the study of social pragmatics. This paper attempts to combine metaphysics with second language acquisition to explore how language modality is copied and disseminated in second language acquisition. I hope that this study of foreign language teachers have some inspiration.

\section{Interpretation of Second Language Acquisition from the Perspective of Masters}

Second language refers to the second language except the mother tongue and second language acquisition (SLA) is the acquisition of the second language except the mother tongue. Second language acquisition is a rule to follow, which requires us to learn in the seemingly complex language phenomenon behind the continuous exploration of its potential laws. As mentioned earlier, we can explore students' second language acquisition from the perspective of memetics. We will be for the second language learners' language learning characteristics, from the perspective of the theory of students to explore the potential of the second language acquisition. We have already mentioned the four stages of memo replication, namely assimilation, memory, expression, transmission. In each of these four stages, some of the memes are eliminated and the four stages are round and round. Similarly, the second language acquisition process also includes four stages, namely, input, memory, output, interaction of these four stages. From the second language acquisition process, the second language knowledge in the form of memes infected learners, into the learner memory, and by the learners attention, understanding and acceptance, the formation of input; when learners use the second language, The memo factor is released from the memory storage model, which is transformed from the memory model to the tangible body that the host can perceive, allowing others to sense the shape of the material, thus realizing the propagation of the memes. The ideal situation of second language acquisition is that the learner's understanding and use of the second language is the same as that of the second language native language, so that the second language can successfully infect the learner and obtain the successful reproduction and dissemination. In the process of second language acquisition, the language model will take two ways, namely, acceptance and exclusion. The language model is replicated and disseminated by establishing an association with the existing knowledge of the second language learner. Sometimes the language model will also be with the original knowledge through the way to achieve the modality of the copy and spread or into other people's brains. The competition process of various memes constitutes the acquisition process and the process of the second language. From this we can conclude that second language acquisition is the process of infection, reproduction and transmission of language. At the same time, it is also the process of competing with the original knowledge of the learners' minds. Thus, the process of second language acquisition is not immutable but full of change. It is important to note that the learner's decoding and the infected phase are the most important processes of meme propagation.

In the process of second language acquisition, there are some variations in the language model during the process of replication propagation. The second language infects the learner in the form of a meme, which requires it to find the closest modality in the original knowledge of the second language learner, thus establishing the association of the two. We can say that the process of second language acquisition is associated with the existing knowledge of the cause of the process. Learners acquire the new knowledge by acquiring the way they have knowledge, so that they can acquire language patterns quickly. Learners in this connection is often considered to establish their own second language memes, in fact, this is a mistake. In this case, due to changes in the extension and connotation of the second language model has long been the language itself has a meme. This kind of primitive knowledge is on the basis of the origin of the variants often leads to rigid phenomenon. The emergence of this problem requires the second language learners to draw high attention and the second language acquisition model and the learner's original knowledge must be different. Only the second language learners often look at their own knowledge of the acquisition and dissemination process, in the use of language to avoid unnecessary pragmatic mistakes, so flexible and comfortable. 


\section{The Enlightenment to Foreign Language Teaching}

Through the above analysis, we can find that modalism plays a decisive role in the process of second language acquisition. Therefore, teachers should be modal theory and students of the second language acquisition process combined in the teaching of conscious inspiration students. This requires teachers to inspire second language learners to focus on imitation at the initial stage of learning the language. In the initial stage of learning, in addition to imitation also need to increase the amount of reading, the formation of language knowledge of precipitation and reserves, that is, a large number of language input; this requires teachers in the teaching process to help students master a large number of phrases, fixed collocation and sentence And so on, that is, to help students find the language of success and make these templates continue to be copied. This stage will help students to gain a better understanding of the second language and make them more in-depth understanding of the language. After which they may be like the language taught by teachers and learn to appreciate the beauty of language. It is possible for these precipitated linguistic knowledge to infect learners in the form of memes, so that learners can use the second language to release the memes from the memory modules to achieve the spread of memes, The output of two languages. In addition, teachers should also inspire students not only in accordance with the language model of the mode of transmission to learn to copy the contents of the study, but also learn to integrate and innovate the language.

\section{The Memetics Impact on College English Teaching}

From the perspective of math theory to look at second language acquisition, then it is the second language in the second language learners learning process has been copied, repeated emulation, and ultimately the use of the purpose of the use of second language exchange. The second language of learning is the language of the meme, including words, sentences, paragraphs, chapters or grammar, habitual expression, etc.; and these two language model is not a complete copy of the second language learners in the Under the guidance of the teacher, under the influence of their own subjective initiative to learn to master a specific language memes, with a certain purpose, language model in this process to achieve replication, imitation and reorganization. Therefore, the modal theory of second language acquisition has a certain guiding significance and research significance. The input and output of the phantom is the two important stages of copying and propagating the meme. In the following, the author mainly introduces the input of the second language and the inspiration of the output. In order to be copied and disseminated, the second language learner must first have full language input, as much as possible to contact the language, the general language input to listen and read the expression, so that the language model can be successful Infection on the learner, into his memory, to develop into the host; the other hand, the meme is only expressed by the host is likely to be spread, reflected in the language model, that is, learners should be as much The use of the language, whether it is written or written. The reproduction, reorganization, and dissemination of the two-memorials are also the need for a means of transmission or a medium through which the "infection and boarding of the second language learners" can be completed, and the various objective conditions of the communication process Together constitute the background and environment of its communication, and only a good communication environment to ensure adequate and effective language mode input and high-quality output. Input and output of the two processes, a good two-language environment is necessary Indispensable condition.

\section{Conclusion}

Language itself is a kind of meme, language phenomenon has aroused more and more attention in linguistics and it has an important guiding significance for second language acquisition, not only in the environment and the role of teachers in two aspects. There are still deep things waiting for mining and play its role in the second language teaching work and inspiration. 


\section{References}

[1] Zhao Gengmei, Wang Xiaobin. Study on the spread of meme and buzzwords [J]. Journal of Xi'an International Studies University, 2007 (02):12-14

[2] Xie Zhaqun, He Nisan, Susan Blackmore. Misunderstanding of the meme - Discuss with Mr. Liu Yuhong [J]. Foreign Language Teaching, 2007 (03):51-55

[3] Xie Zhuoqun, He Nong. Language Temperament theory[J]. Modern Foreign Language, 2007 (01):62-63

[4] Chen Linxia. Modes in advertising language[J]. Foreign Language Teaching, 2006 (04):110-111

[5] He Nong. Language in the language[J]. Language Science, 2005 (06):25-28

[6] Chen Yan. Language input and output theory on foreign language classroom teaching enlightenment [J]. Contemporary Educational Science, 2003 (17):42-46 